\title{
AN EFFICIENT APPROACH ON SPATIAL BIG DATA RELATED TO WIRELESS NETWORKS AND ITS APPLICATIONS
}

\author{
Sowmiyaa $\mathbf{P}^{1}$, Priyadharshini $\mathbf{V}^{2}$, Minojini $\mathbf{N}^{3}$, Gayathri R Krishna ${ }^{4}$ \\ ${ }^{1} P G$ Scholar, Computer Science and Engineering, Dr.N.G.P Institute of Technology, Tamilnadu, India \\ ${ }^{2} P G$ Scholar, Computer Science and Engineering, Dr.N.G.P Institute of Technology, Tamilnadu, India \\ ${ }^{3} P G$ Scholar, Computer Science and Engineering, Dr.N.G.P Institute of Technology, Tamilnadu, India \\ ${ }^{4}$ PG Scholar, Computer Science and Engineering, Dr.N.G.P Institute of Technology, Tamilnadu, India
}

\begin{abstract}
Spatial big data acts as a important key role in wireless networks applications. In that spatial and spatio temporal problems contains the distinct role in big data and it's compared to common relational problems. If we are solving those problems means describing the three applications for spatial big data. In each applications imposing the specific design and we are developing our work on highly scalable parallel processing for spatial big data in Hadoop frameworks by using map reduce computational model. Our results show that enables highly scalable implementations of algorithms using Hadoop for the purpose of spatial data processing problems. Inspite of developing these implementations requires specialized knowledge and user friendly.
\end{abstract}

Keywords: Spatial Big Data, Hadoop, Wireless Networks, Map reduce

\section{INTRODUCTION}

Proliferation of measurement capabilities in wireless nodes is making a deluge of knowledge that may be harvested from wireless networks. Smartphone and other mobile terminals are presently capable of activity large numbers of various properties of the encircling environment, and additional data performance of the networks themselves is changing into available through new operation interfaces. Today mobile phones are moving and distributed everywhere is likely to form them a dominant supply of sensing data either directly from the sensors that may be embedded into the terminal instrumentation or as a mobile entry that works as a data relay for alternative sensing devices. Moreover, wireless sensor networks and additional typically the event of the Internet of Things (IoT) applications is more extending the realm of measurement information that may be accessed wirelessly. Processing of this large quantity of knowledge is clearly a key challenge for future applications, almost like alternative "big data" developments in numerous analysis domains [1].

In this article we have a tendency to argue that the world of huge knowledge within the context of wireless networks may be a difficult and exciting analysis topic on its own, and additionally has its own somewhat distinct issues. There are lots of areas; particularly one considers offline analysis of information that originates from wireless networks. Hence, existing huge knowledge applications in wireless networks furthermore have centered on relative knowledge (i.e., mining relationships from classical databases that square measure generated from wireless data). However, we have a tendency to note that an outsized portion of data from wireless networks is inherently spacial and spatio-temporal (i.e., it's position-dependent and infrequently exhibits sturdy spacial correlations). Collection, storage, and mining of such knowledge have their own challenges and peculiarities that don't continuously align with the on-line database approach. In spacial huge knowledge issues we have a tendency to square measure seeking to perform inference on giant amounts of measurements that square measure associated to specific locations and time instances those measurements were conducted at. The ensuing estimation issues are terribly distinct from classical data processing ones, motion terribly different quantifiability challenges, furthermore as implementation requirements. For instance, by currently many algorithms exist to perform classification and prediction on relative knowledge in linear time, whereas classical spacial prediction algorithms require isometric time within the variety of obtainable measurements [2]. Clearly this presents a huge quantifiability gap that must be closed before true huge spacial knowledge applications in wireless networks become possible.

This article has 2 objectives. We have a tendency to initial gift 3 categories of application situations within which spatio-temporal massive knowledge plays a distinguished role, outlining the categories of knowledge offered, the origins of the massive knowledge, and also the objectives of its process. The first part of the article we have a tendency to hope to offer the reader AN understanding of a number of the foremost relevant application situations. We then discuss a number of our own analysis add this domain toward developing and implementing solutions for wireless spatial big processing. Here we have a tendency to emphasize the necessity to own both smart recursive solutions yet because they ought to develop suitable implementation frameworks that alter those algorithms to run on progressive procedure clusters. 


\section{APPLICATION FOR SPATIAL BIG DATA}

In this section we have a tendency to describe in additional detail 3 example eventualities for process abstraction information from wireless networks, and explain why huge information issues arise here. Every of those application scenarios has been designated for example a selected key challenge they cause for abstraction huge processing in wireless networks.

\subsection{Management of Wireless Networks Using Big}

\section{Data}

One of the main trends within the development of wireless network architectures has been the many increase in their instrumentation. In such as the step-down of drive tests (MDT) in cellular networks, combined with enhanced measurement capabilities [3] across the protocol stack in mobile phones and wireless fidelity devices, are enabling network operators to reap new amounts of knowledge from their networks [4]. The information may be mined to optimize networks, increase security through anomaly detection, and supply and enhance application behavior, among alternative things. The key challenge here is the way to fuse this knowledge in associate degree best manner for deciding, optimization, and network operation.

Currently used approaches for estimating the coverage of a wireless network embrace elaborate propagation simulations drive tests. However, these will solely provides a rough estimate of network coverage at the best. Propagation simulations have inherent inaccuracies owing to restricted details on the out there building and landscape information, and drive check area unit terribly expensive to conduct; especially, urban area unites are dynamic enough that conditions can modification throughout the lifespan of the network preparation. a pretty various is to use mobile terminals to enhance the drive and it giving the operator an outsized range of path loss or received signal strength (power) measurements and estimates in conjunction with the GPS locations wherever they were measured. Supported these measurements, the operator would then prefer to perform special estimation or spacial interpolation so as to get a coverage map for the complete region of interest. Constant information will also be accustomed perceive interference and usage patterns of the network underneath study. Such approaches area unit vital for operators managing the wireless networks and further on improve being in dynamic spectrum such as for mistreatment TV white areas for cellular and wireless fidelity styles of use. Such associate degree approach, firmly grounded on nearreal-time process of activity information from millions or tens of millions of terminals, would considerably scale back the time to sight and localize coverage holes and abnormal interference sources, and perform alternative network diagnostic tasks. However, classical data processing algorithms for giant information issues cannot obtain such spacial estimates. Currently, wide used special interpolation ways, like non-parallelized kriging implementations and compressive sensing and also have poor measurability properties, severely limiting the number of data that may be handled in an exceedingly given region.

\subsection{Massive Sensing and IoT Applications}

Another major trend within the wireless communications domain has been the maturing of wireless device networking (WSN) research, recently being subsumed underneath the generic term of Internet of Things (IoT) [5]. Existing work on WSNs, especially in the analysis community, has had a powerful prototyping focus, which has resulted in smart understanding of the sensible implementation challenges, however has conjointly considerably restricted the size of deployments. Even 1000-node networks are thought-about as terribly large-scale systems, whereas victimization smartphones, vehicles with communications and activity capabilities, and large-scale IoT infrastructures may end up in millions or perhaps billions of device readings with timespace categorization inside one city. Clearly, the information process challenges that emerge square measure on a very totally different scale compared to existing WSN trials.

Many of the info process challenges for this application scenario square measure clearly almost like the wireless network management case above. However, there also are some key variations, which is why we tend to believe this case ought to be seen as a distinct one. First, several of the info sources have terribly restricted computational capabilities, and attributable to the inherent want for the hardware platforms to be low cost to supply, even have restricted memory, clock accuracy, and different shortcomings that require to be restrained within the part. Second, the scales concerned square measure even larger if widespread deployments of measurement-capable IoT platforms become a reality. Third, connectivity to the backend infrastructure may well be periodic in nature and have confidence technologies like knowledge mulling through delay tolerant networks (DTNs) of terribly restricted turnout. One risk is to use mobile phones as relay or knowledge mule devices. However, the sheer quantity of information in addition as economic concerns mean those mobile phones cannot act as dumb relays that directly forward knowledge while not some process or introducing delays. These limitations may necessitate a lot of more knowledge aggregation and preprocessing on an area level (as opposed to the operator back-end within the initial case).

\subsection{Smart Cities and Noisy Spatial Data}

The key options of the previous 2 application eventualities have been the increasing scale of the obtainable knowledge, as well as the diversity of procedure resources. However, both of them have centered on manufacturing estimates, usually for human operators in a very single vertical sector of the trade. Our third application state of affairs relaxes the last condition.

In applications like exploitation large-scale sensing to drive automatic functions in sensible cities, 2 new challenges emerge [6-8]. The primary of those is use of information for time period control selections, as an example, for rerouting of traffic flows and public transportation programming supported coordinate system process of traffic knowledge, pollution estimates, and noise levels. All of this data will 
without delay be obtained from existing platforms, and data assortment is changing into easier and easier; so, the challenge is that of study enough process of information in a very sufficiently speedy manner to change real time control applications. We note, as an example, that the traffic planning and observance is supported aggregation locations of mobile phones on a colossal scale. This will be done entirely anonymized means, during which the cellular network data is used while not user identities to observe movement patterns. This imposes significantly rigorous conditions on the standard of the estimates from processing and illation, as well as the requirement for the algorithms concerned to estimate the reliability of the estimates they manufacture.

The second distinctive facet of this application situation is that the need to cross-correlate varied knowledge sources for getting the best and most sturdy estimates. for instance, as hinted at above, traffic management selections normally would be made supported variety of call variables, like level of congestion on roads, noise levels elicited, interaction with public transport, pollution caused, and so on. Such multisensory data processing and call rules are extraordinarily difficult to design in top-down fashion, and instead would require scalable machine learning techniques, robust prediction parts, finding seasonality and different periodic patterns in large knowledge sets, tools for anomaly detection, and isolation of causal relations from knowledge.

\section{SELF-AWARENESS OF ERRORS}

Algorithms that not solely yield estimates, however additionally confidence intervals for these estimates, are rather more helpful than adhoc predictors that don't provide steering on their expected performance.

\subsection{Match with Computational Infrastructures}

There is a robust convergence toward a low range of machine infrastructures, generally enforced as cloud services, enabling straightforward parallelization and computation on massive data sets. Algorithms which will be enforced on such infrastructures are inherently a lot of applicable for several applications than those requiring in depth "impedance matching" between the rule and also the underlying machine infrastructure.

\subsection{Extendibility with Prior and Side Information}

In several cases estimation issues are 1st solved with one type of information solely, and therefore the algorithms are tailored for this one type of information. This typically creates surplus challenges to incorporate extra data or previous data to the decisions created if such data becomes out there later on. Thus, algorithms that may be extended toward a full Bayesian reasoning framework and toward variable settings should be most well-liked over univariate fixed-parameter variants whenever possible.

\subsection{Controlled Approximations}

Finally, we note that many exact inference procedures have computational complexities that are too high for big data applications, but also enable linear-time approximations with optimality guarantees. Such algorithms are extremely powerful since they allow the computational burden to be controlled based on the amount of data available.

\subsection{Scalable Computational Solutions}

In the past number of years our cluster has conjointly done intensive implementation work on abstraction and spatiotemporal estimation algorithms based on trendy cloud. We tend to discuss concisely the key lessons learned from these prototype implementations, linking our experiences to the desirable rule properties mentioned higher than.

We focus here on spatial estimation or spatial interpolation using totally different variants of the kriging algorithmic rule [2] as a basis for our work. Kriging may be a key example of AN algorithmic rule that satisfies the properties mentioned above; that's, providing best and study spatial estimates, and enabling theorem extensions also as incontrovertibly best linear-time approximations [9-11].

Our objective is to specifically an extremely ascendable parallelizable implementation of kriging supported the Hadoop [12], HBase [13], and Map Reduce machine framework. We also, providing a flexible framework on that totally different applications for process of data obtained from WSNs and alternative measuring sources will be enforced. For a lot of technical details on the implementation design beside further performance results [14].

As a basic underlying resource we have a tendency to outline an info (DB) schema consisting of a set of tables for storing individual sensor readings, moreover as any data on the networks themselves, like estimates of accuracies of the sensors used. These are all enforced exploitation HBase because the storage framework. On high of this basic assortment of tables, further information models are outlined for analyzing and modeling sensors. These estimates are also keeping in HBase employing custom information.

The key computations concerned within the kriging rule are the estimation of a correlation live known as the semivariogram from the measurements, and exploitation this correlation measure to construct a matrix. The inverse of this correlation matrix will then be employed in computing optimum spacial predictions of the phenomena of interest (e.g., received signal strength or noise and pollution levels) supported the available measurements. The interested reader will realize the mathematical description. The typical structure of the experimental semivariogram and it is half of the variance of measurements conducted at distance apart, is shown for a typical information set, beside a fitted semivariogram model that enables constant modeling and estimation of the matrix. 
For computing the semivariogram we'd like to search out all node pairs at a given distance and also calculate the variance of their various detector readings. This leads to a measure of correlation or dispersion of the values as a perform of the space, which might be sculptured by a straightforward constant function and utilized in the prediction task. The key machine bottleneck during this method is finding all node pairs at a given distance, since naïve implementation gazing all node pairs takes quadratic time, introducing a severe machine bottleneck. Our implementation overcomes this bottleneck by using KD-trees for considerably dashing up this search and enabling a high degree of similarity within the implementation enabled by the Map Reduce machine model.

It consisting of modules known as DatabaseM, Database2dtreeM, Calculated SemivariogramM, and Analytical Semivariogram, won't to get the required correlation measure. The abstraction prediction exploitation the semivariogram model is then achieved by the module spatial predictionM also in parallel fashion. We have a tendency to in short describe below the implementation details of every module. Just in case of parallel processed module, we have a tendency to outline the input and output values for the map and reduce functions inherent to the Map Reduce machine model. we have a tendency to highlight additionally the mathematical calculations complete in the clerk and also the ones complete within the reducer.

The DatabaseM module merely writes the sensing element information in Associate in Nursing HBase info. It's a Map Reduce Java program implementing a mapper. The input price of the map operate is that the source of domestically accessible sensing element information. The module launches several mappers reading the info from the info sources, converting the data formats as required, and writing them into a specific HBase.

Database2d-treeM may be a easy Java category having 2 main functions: a builder and an enquiry perform. The builder reads the locations of the nodes from the info and inserts them into a $2 \mathrm{~d}$-tree referred to as 2 dtreeDB. The search perform identifies the nodes settled during a predefined geographical region sanctioning the quick computation of the required semivariogram.

CalculatedSemivariogramM may be a Map Reduce program implementing a clerk and a reducer. Its major job is computing the experimental semivariogram (i.e., variance of sensing element readings from all nodes separated by a given distance $h$ ). For a specified $h$, the clerk searches within the 2dtreeDB all the nodes falling into the correspondent bin, and calculates the variance of the corresponding sensing element readings. Upon reception of all the variances, the reducer computes the ultimate worth of the semivariogram as a perform of the variances over the separation distance $h$.
The final steps within the abstraction estimation method square measure the fitting of the constant quantity model into the obtained semivariogram estimate, and victimization that to reckon the matrix and the final abstraction estimate. The fitting is finished victimization AN implementation within the Analytical SemivariogramM module. The latter is an easy Java category victimization the web mathematical functions offered by the core Hadoop framework. The Spatial predictionM module is finally answerable for computing the abstraction estimate. It's additionally a Map Reduce program implementing a mapper and a reducer. The map operate constructs the matrices concerned within the prediction task in row-wise fashion, and the scale back operate constructs the complete matrices supported these partial inputs and interpolates the required abstraction price.

\section{DEPLOYMENT PLANNING AND}

\section{HIERARCHICAL NETWORK DIMENSIONING}

Large-scale WSN and IoT deployments[15] may result in very massive information streams, far exceeding most presently used net applications and processing solutions are required to influence such immense quantities of knowledge, and coming up with such deployments collectively with the info process framework may be a difficult task that new improvement solutions are needed and completely different aspects of price, robustness, performance, and latency kind advanced trade-offs for such coming up with tasks. For example, adding new information fusion and process centers even once not strictly required in terms of machine power will increase readying prices, however at the same time considerably improve hardiness (by removing single points of failure) and cut back latency.

\section{SOFTWARE FRAMEWORKS IMPLEMENTATION LANGUAGES}

More and a lot of totally different process models area unit rising with new information back-end sorts. Area unit a number of them a lot of appropriate for these sorts of computations than Map Reduce types of solutions. There's clearly a desire to balance totally different implementation objectives from the performance of the system to fault tolerance between computation and storage, and so on. New proposals for programming languages also are rising, and it's not clear which of them ought to be used as a foundation for future data processing solutions. As for runtime implementation frameworks, the trade-offs between implementation and runtime complexities area unit key problems for the underlying programming languages in addition.

\section{CONCLUSION}

It is a key role for future wireless networking applications. While it's a definite class from relative massive information, we have conjointly shown through our implementation examples that existing parallel processing and process frameworks, like HBase and Hadoop. However, care should be taken within the style of the implementation design to 
learn from the high degree of similarity enabled by Hadoop. From our implementation experiences in addition as from the analysis of the 3 given application situations, we believe there's a robust demand at intervals the wireless community for a typical platform for storage and processing of spatial and spatio-temporal information. This platform would possibly preferably be based on Hadoop and HBase styles. But should offer a considerably higher level of abstraction to users. In our case vital experience on each the implementation details of the Map Reduce model and therefore the underlying spatial statistics algorithms was required, far more therefore than a typical user of big data processing frameworks can be expected to have in the future. Dedicated frameworks like varied geographic information systems (GISs) have a number of these

Options. However, they're presently extremely specialized for specific inference tasks, typically have restricted measurability, and infrequently provide a flexible programming model for developing new processing applications. We tend to see the development of a new and more powerful various to these tools as the key challenge for the longer term.

\section{REFERENCES}

[1]. C. Lynch, "Big Data: How Do Your Data Grow?," Nature 455, no. 7209, 2008, pp. 28-29.

[2]. N. Cressie, Statistics for Spatial Data, 2nd ed., Wiley, 1993.

[3]. B. Sayrac et al., "Cognitive Radio Systems Specific for IMT Systems: Operator's View and Perspectives," Telecommun. Policy, 2013.

[4]. W. Hapsari et al., "Minimization of Drive Tests Solution in 3GPP," IEEE Commun. Mag., vol. 50, no. 6, 2012, pp. 28-36.

[5]. L. Mainetti, L. Patrono, and A. Vilei, "Evolution of Wireless Sensor Networks Towards the Internet of Things: A survey," Proc. 19th Int'l. Conf. Software, Telecommun. and Computer Networks, 2011, pp. 1-6.

[6]. D. Cuff, M. Hansen, and J. Kang, "Urban Sensing: Out of the Woods," Commun. ACM, vol. 51, no. 3, 2008, pp. 24-33.

[7]. P. Dutta et al., "Common Sense: Participatory Urban Sensing Using a Network of Handheld Air Quality Monitors," Proc. 7th ACM Conf. Embedded Networked Sensor Systems, 2009, pp. 349-50.

[8]. M. Naphade et al., "Smarter Cities and Their Innovation Challenges," Computer, vol. 44, no. 6, 2009, pp. 32-39.

[9]. B. Sayrac et al, "Improving Coverage Estimation for Cellular Networks with spatial Bayesian Prediction based on Measurements," Proc. 2012 ACM SIGCOMM Wksp. Cellular Networks: Operations, Challenges, and Future Design, 2012, pp. 43-48.

[10]. N. Cressie and G. Johannesson, "Fixed Rank Kriging for Very Large Spatial Data Sets," J. Royal Statistical Society: Series B (Statistical Methodology), vol. 70, no. 1, 2008, pp. 209-26.
[11]. J. Riihijärvi, J. Nasreddine, and P. Mähönen, "Demonstrating Radio Environment Map Construction From Massive Data Sets," Proc. IEEE DYSPAN'12, 2012, pp. 266-67.

[12]. Hadoop, http://hadoop.apache.org/, Dec. 2013.

[13]. HBase, http://hadoop.apache.org/hbase/, Dec. 2013.

[14]. C. Jardak et al., "Parallel Processing of Data from Very Large-Scale Wireless Sensor Networks," Proc. 19th ACM Int'l. Symp. High Performance Distributed Computing, 2010, pp. 787-94.

[15]. C. Jardak, J. Riihijärvi, and P. Mähönen, "Extremely Large-Scale Sensing Applications for Planetary WSNs," Proc. 2nd ACM Int'l. Wksp. Hot Topics in Planet-Scale Measurement, 2010, p. 3. 